\title{
Impacto dos grandes estudos multicêntricos sobre a prática obstetrtica
}

\author{
Impact of large multicenter studies on obstetrical practice
}

Nelson Sass

Apesar dos esforços dispendidos para redução de problemas, os indicadores atuais relativos à saúde materna e perinatal em nosso país sinalizam para a necessidade da ampliação de estratégias que resultem em efetiva oferta de qualidade da assistência ${ }^{1}$. A segurança técnica oferecida às pacientes não se esgota na disponibilidade de recursos técnico-administrativos, mas também pela capacitação da equipe assistencial em solucionar problemas muitas vezes críticos. Mas o que têm a ver estudos multicêntricos com nossos problemas e com nossa prática obstétrica?

A resposta nos remete para a necessidade diária da tomada de decisões frente a inúmeras questões clínicas e a incerteza dos resultados a partir das intervenções preconizadas. Para um determinado problema, muitas vezes existem propostas diversas, eventualmente com riscos diferentes, sem que tenham sido ponderados de maneira consistente os riscos e os benefícios entre as opções. Tais questões são mais relevantes ainda quando nos deparamos com novas alternativas, as quais confrontam formas de tratamento tradicionais. Para todas estas perguntas, as melhores respostas e, portanto, os melhores resultados do ponto de vista das pacientes devem ter origem em estudos controlados, nos quais os grupos incluídos tenham sido escolhidos de forma aleatória, com tamanho amostral representativo e suficiente para detectar diferenças através de análises estatísticas apropriadas ${ }^{2,3}$.

Dentro deste cenário, a Epidemiologia Clínica tem procurado auxiliar as tomadas de decisões sobre os cuidados individuais em saúde, tendo o compromisso com a associação da experiência do médico com as melhores evidências cientificas disponíveis na literatura. Neste sentido, acreditamos ser importante louvar as iniciativas do Ministério da Saúde apoiadas pela FEBRASGO e cristalizadas através de publicações importantes como o manual técnico "Parto, Aborto e Puerpério. Assistência Humanizada à Mulher”, que destaca a importância da atuação médica compromissada com decisões baseadas em evidências e sintonizada com novas propostas e experiências, resultando em práticas saudáveis e seguras. Nesta mesma linha, também devem ser destacados os Seminários Nacionais sobre Assistência Obstétrica e Neonatal Humanizada Baseada em Evidências Científicas ${ }^{5}$, desenvolvidos para sensibilizar e capacitar instituições selecionadas em todos os estados brasileiros, a partir de sua relevância local na assistência materna e perinatal.

Em relação à formação da opinião médica, é importante também destacar que, durante muito tempo, as decisões clínicas foram baseadas nas experiências pessoais, nas opiniões dos indivíduos com mais autoridade e nas teorias fisiopatológicas. O questionamento crescente acerca da real efetividade das intervenções preconizadas por muito tempo desencadeou um processo de aferição destas recomendações através de provas científicas rigorosas, reduzindo a ênfase à prática não sistematizada e às teorias fisiopatológicas, para se concentrar na análise apurada dos métodos pelos quais as informações médicas foram e serão obtidas, em especial ao desenho da pesquisa, sua condução e a análise estatística.

Frente a uma verdadeira avalanche de informações que se avoluma com velocidade espantosa, é praticamente impossivel ter acesso a toda a publicação médica em tempo oportuno, gerando assim a necessidade de se explorar o conhecimento utilizando-se de métodos bem definidos para avaliação crítica, preferencialmente a partir de revisões sistemáticas da literatura médica e do bom senso, para adaptar esses conhecimentos às condições de cada paciente e ao contexto da realidade sócio-econômica local. A principal matéria prima para a construção de revisões sistemáticas com conclusões consistentes é gerada a partir de ensaios clínicos com grande casuística. Tendo em vista a urgência para estas respostas, a saída mais plausivel é o desenvolvimento de estudos multicêntricos que permitam somar esforços para inclusão de pacientes em número suficiente para compor tamanho amostral adequado no menor espaço de tempo possivel, integrando as informações de forma crítica e com maior poder estatístico para detectar possiveis diferenças entre grupos expostos, reduzindo ao máximo a incerteza das decisões clínicas.

Verificamos que, felizmente, esta metodologia tem se ampliado, uma vez que inúmeros ensaios, em diversas especialidades médicas, foram, estão sendo e serão desenvolvidos, tendo em comum a busca de respostas apropriadas e honestas para perguntas ainda em aberto sobre terapia, prognóstico, profilaxia

Professor Adjunto do Departamento de Obstetrícia da Universidade Federal de São Paulo - UNIFESP - São Paulo (SP), Brasil. 
e relação custo-benefício. Importante ainda ressaltar que, muitas vezes, mesmo revisões sistemáticas são incapazes de fornecer conclusões consistentes sobre determinado problema em face do pequeno número de trabalhos clínicos selecionados e do número de pacientes incluídos. Para estas situações, torna-se necessário o desenvolvimento de ensaios clínicos adicionais com metodologia adequada a fim de permitir conclusões mais consistentes.

Para que se entenda como um ensaio pode mudar a prática clínica, ilustramos esta questão utilizando um exemplo importante relativo aos problemas afeitos às sindromes hipertensivas da gravidez: o Magpie trial $^{6}$. Este grande ensaio multicêntrico teve como objetivo elucidar a seguinte dúvida: é de conhecimento geral que o sulfato de magnésio é a melhor alternativa para o controle de pacientes com eclâmpsia (esta informação é originada de ensaios multicêntricos), porém teria o sulfato de magnésio uma ação preventiva para a ocorrência de eclâmpsia? Mesmo em revisão sistemática disponivel sobre o tema ${ }^{7}$, esta questão não encontrava resposta adequada. Após a inclusão de cerca de 10 mil pacientes, os resultados do Magpie identificaram que o uso do sulfato de magnésio reduziu em mais de $50 \%$ o risco de eclâmpsia em mulheres com pré-eclâmpsia. A combinação dos resultados do Magpie com os dos estudos incluídos na revisão sistemática ${ }^{7}$ identificou uma redução de $59 \%$ do risco de uma mulher com pré-eclampsia apresentar convulsão quando comparado com placebo (RR: 0,41; IC 95\%: 0,29-0,58). A partir de então, o uso de sulfato de magnésio para estas situações deve ser recomendado baseado em evidências muito consistentes.

Além da importância dos resultados obtidos em ensaios multicêntricos, cumpre destacar a presença brasileira não só neste ensaio clínico como em muitos outros como o "Eclampsia trial's e o $\mathrm{ECCPA}^{9}$, entre muitos outros. Este aspecto destaca em nosso país o papel importante de vários centros responsáveis pela assistência, ensino e pesquisa de nossa especialidade, sendo que o intercâmbio com organizações internacionais tem sido benéfico pela incorporação de habilidades que poderão facilitar o desenvolvimento de trabalhos cooperativos nacionais.

Existem centros de excelência em todo o País que produzem informações de muita qualidade, mas é quase certo que esbarram nas dificuldades para a elaboração de projetos mais amplos. A união de esforços e de talentos pode gerar uma oportunidade impar para alavancar nosso conhecimento e também para criar impacto junto às autoridades responsáveis pela implantação de politicas de saúde. O trabalho cooperativo multicêntrico poderá produzir, em tempo relativamente curto, informação de qualidade que possa mais rapidamente ser incorporada em nossa prática clínica.

\section{Referências}

1. Alencar Júnior CA. Os elevados índices de mortalidade materna no Brasil: razões para sua permanência. Rev Bras Ginecol Obstet. 2006;28(7):377-9.

2. Greenhalgh T. How to read a paper. The basics of evidence based medicine. 2nd ed. London: BMJ Books; 2001.

3. Sackett DL. The principles behind the tatics of performing therapeutic trials. In: Haynes RB, Sackett DL, Guyatt GH, Tugwell P, editors. Clinical epidemiology. How to do clinical practice research. 3rd ed. Philadelphia: Lippincot Williams \& Wilkins; 2006. p. 173-243.

4. Brasil.Ministério da Saúde. Secretaria de Políticas de Saúde. Área Técnica de Saúde da Mulher. Parto, aborto e puerpério. Assistência humanizada à mulher. Brasília: Ministério da Saúde; 2001.

5. Brasil. Ministério da Saúde. Secretaria de Atenção à Saúde. Área Técnica de Saúde da Mulher. Anais dos seminários nacionais sobre assistência obstétrica e neonatal humanizada baseada em evidências. Brasília: Ministério da Saúde; 2005.

6. Altman D, Carroli G, Duley L, Farrell B, Moodley J, Neilson J, et al. Do women with pre-eclampsia, and their babies, benefit from magnesium sulphate? The Magpie Trial: a randomised placebo controlled trial. Lancet. 2002;359(9321):1877-90.

7. Duley L, Gülmezoglu AM, Henderson-Smart DJ. Magnesium sulphate and other anticonvulsants for women with pre-eclampsia. Cochrane Database Syst Rev. 2006;(1):CD000025.

8. Duley L. Magnesium sulphate regimens for women with eclampsia: messages from the Collaborative Eclampsia Trial. Br J Obstet Gynaecol. 1996;103(2):103-5.

9. ECPPA: randomised trial of low dose aspirin for the prevention of maternal and fetal complications in high risk pregnancies. pregnant women. ECPPA (Estudo Colaborativo para Prevenção da Pre-eclampsia com Aspirina) Collaborative Group. Br J Obstet Gynaecol. 1996;103(1):39-47. 